\title{
Taxonomic novelties published in Folia Cryptogamica Estonica, fascicles 1-50
}

\author{
Andres Saag \\ Institute of Ecology and Earth Sciences, University of Tartu, Lai St. 38/40, 51005 Tartu, Estonia. \\ E-mail: andres.saag@ut.ee
}

Newly described taxa (in bold) or new combinations, altogether 237 taxonomic novelties which have been published in Folia Cryptogamica Estonica (FCE), fascicles 1-50 (1972-2013), are listed in alphabetical order, together with their publication data. The following marks are used for indicating some major taxonomical or ecological groups of treated taxa:

$\S$ - bryophytes;

+- non-lichenized fungi

* - lichenized fungi;

\# - lichenicolous fungi.

+ Agaricus bisporus (Lange) Imbach var. albidus (Lange) Singer f. microspora Kalamees FCE 15: 6 (1981).

+ Agaricus luteoflocculosus Kalamees - FCE 17: 1 (1985).

+ Agaricus subsquamuliferus Kalamees FCE 27: 5 (1989).

+ Albotrichia kamtschatica (Raitv.) Raitv. - FCE 17: 3 (1985).

+ Albotrichia kurilensis Raitv. - FCE 2: 14 (4 Jan 1973).

+ Albotrichia longispora Raitv. - FCE 2: 15 (4 Jan 1973).

+ Albotrichia minuta Raitv. - FCE 2: 14 (4 Jan 1973).

+ Albotrichia pallida Raitv. - FCE 2: 14 (4 Jan 1973).

+ Albotrichia vantschensis Raitv. - FCE 12: 1 (1981).

* Arctocetraria simmonsii (Krog) E.S. Hansen FCE 43: 6 (2007).

* Asahinea culbersoniorum Trass - FCE 29: 31 (1992).

* Aspicilia bennettii (Lynge) Alstrup \& E.S. Hansen - FCE 37: 5 (2001) [2000].

* Aspicilia expansa (Lynge) Alstrup \& E.S. Hansen - FCE 37: 5 (2001) [2000].

+ Asterodontaceae Parmasto - FCE 37: 55 (2001) [2000].

+ Belonidium litorale Raitv. - FCE 13: 8 (1981).

+ Belonidium sacchalinensis Raitv. - FCE 9: 2 (1977).
+ Belonidium sulphurellum (Peck) Raitv. \& Leenurm - FCE 37: 88 (2001) [2000].

+ Belonidium virtembergense (Matheis) Raitv. \& Leenurm - FCE 37: 89 (2001) [2000].

+ Belonidium tianschanicum Raitv. - FCE 12: 2 (1981).

* Biatorella contigua N.S. Golubk. \& Piin FCE 7: 1 (1977).

* Caloplaca austroatlantica Øvstedal - FCE 47: 44 (2010).

* Caloplaca elaeophora (E.S. Hansen, Poelt \& Søchting) Alstrup \& E. S. Hansen - FCE 37: 7 (2001) [2000].

* Caloplaca kiewkaensis Yakovczenko, Galanina \& S.Y. Kondr. - FCE 48: 17 (2011).

* Caloplaca letrouitioides S.Y. Kondr., Elix \& Kärnefelt - FCE 48: 19 (2011).

* Caloplaca mallacootensis S. Y. Kondr. \& Kärnefelt - FCE 50: 41 (2013).

* Caloplaca montenegrensis S. Y. Kondr. \& Kärnefelt - FCE 50: 42 (2013).

* Caloplaca rexii S. Y. Kondr. \& Kärnefelt FCE 50: 44 (2013).

* Caloplaca rossii S. Y. Kondr. \& Kärnefelt - FCE 50: 45 (2013).

* Caloplaca subgyalectoides S. Y. Kondr. \& Kärnefelt - FCE 50: 46 (2013).

* Caloplaca trassii Galanina \& S.Y. Kondr. - FCE 48: 20 (2011).

* Caloplaca ussuriensis Oxner, S.Y. Kondr. \& Elix - FCE 48: 21 (2011). 
+ Camarophyllus albidocinereus Kalamees - FCE 27: 2 (1989).

+ Capillipes kalameesii Raitv. - FCE 1: 4 (15 Apr 1972).

+ Ceraceomyces eludens K.H. Larss. - FCE 33: 74 (1998).

+ Ceraceomyces microsporus K.H. Larss. FCE 33: 75 (1998).

+ Ceratosebacina calospora (Bourdot \& Galzin) P. Roberts - FCE 33: 128 (1998).

+ Ceriporia sulphuricolor Bernicchia \& Niemelä - FCE 33: 15 (1998).

+ Chaenothecopsis jaczevskii (Woron.) Titov FCE 32: 129 (1998).

* Chaenothecopsis trassii Titov - FCE 32: 130 (1998).

+ Cheilymenia tervetensis Raitv. \& Vimba FCE 42: 95 (2006).

+ Cistella atra Raitv. - FCE 12: 2 (1981).

+ Cistella flavorubens Raitv. - FCE 12: 2 (1981).

+ Cistella pediformis Raitv. - FCE 12: 3 (1981).

* Cladina beringiana (Ahti) Trass - FCE 1: 3 (5 Apr 1972).

* Cladina ciliata (Stirt.) Trass - FCE 11: 6 (1978).

* Cladina mitis (Sandst.) Hale \& W. L. Culb. f. arenicola Trass - FCE 11: 6 (1978).

* Cladina oxneri (Rass.) Trass - FCE 1: 3 (15 Apr 1972).

* Cladina tenuiformis (Ahti) Trass - FCE 1: 3 (15 Apr 1972).

* Cladonia alinii Trass - FCE 11: 1 (1978).

* Cladonia groenlandica (Å.E. Dahl) Trass-FCE 1: 2 (15 Apr 1972).

* Cladonia macroceras (Flörke) Ahti var. nigripes (Nyl.) Trass - FCE 11: 5 (1978).

* Cladonia nigripes (Nyl.) Trass - FCE 1: 2 (15 Apr 1972).

* Cladonia subrangiformis Sandst. f. spinulifera Trass - FCE 11: 6 (1978).

* Cladonia trassii Ahti - FCE 32: 7 (1998).

* Cladonia uliginosa (Ahti) Ahti - FCE 32: 7 (1998).

+ Cladobotryum gracile K. Põldmaa-FCE34: 24 (1999).

+ Clitocybe subfestiva Kalamees - FCE 26: 3 (1987).

+ Coltricia stuckertiana (Speg.) Rajchenb. \& J.E. Wright. - FCE 33: 119 (1998).

+ Conocybe obliquopora Hauskn. \& Kalamees - FCE 45: 29 (2009).
+ Conocybe semidesertorum Hauskn. \& Kalamees - FCE 45: 32 (2009).

+ Conocybe uralensis Hauskn., Knudsen \& Mukhin - FCE 45: 33 (2009).

+ Coprinus vošoustii Pilát var. steppicola Kalamees - FCE 15: 6 (1981).

+ Dasyscyphus alnifolius Raitv. - FCE 9: 4 (1977).

+ Dasyscyphus altaicus Raitv. - FCE 9: 5 (1977).

+ Dasyscyphus dasiphorae Raitv. - FCE 9: 6 (1977).

+ Dasyscyphus ledi Raitv. - FCE 9: 4 (1977).

+ Dasyscyphus pseudocannabinus Raitv. FCE 9: 6 (1977).

+ Dasyscyphus pudicelloides Raitv. - FCE 9: 5 (1977).

+ Dasyscyphus vantschensis Raitv. - FCE 12: 1 (1981).

+ Dentipellis microspora Y.C. Dai - FCE 33: 25 (1998).

+ Dichochaete Parmasto - FCE 37: 56 (2001) [2000].

+ Dichochaete ceratophora (Job) Parmasto-FCE 37: 56 (2001) [2000].

+ Dichochaete setosa (Sw.) Parmasto - FCE 37: 57 (2001) [2000].

+ Elmerina hexagonoides (A. David \& Jaq.) Núñez [ as 'hexagonioides'] - FCE 33: 100 (1998).

$\S$ Endogemmataceae Konstant., Vilnet \& A.V. Troitsky - FCE 48: 132 (2011).

$\S$ Endogemma Konstant., Vilnet \& A.V. Troitsky - FCE 48: 132 (2011).

$\S$ Endogemma caespiticia (Lindenb.) Konstant., Vilnet \& A.V. Troitsky - FCE 48: 132 (2011).

+ Entoloma subglobispora Kalamees - FCE 27: 4 (1989).

+ Favolaschia sacchalinensis Parmasto FCE 6: 43 (1974).

+ Galerina ovalispora Kalamees - FCE 15: 8 (1981).

* Gyalidea goughensis Øvstedal - FCE 47: 44 (2010).

+ Gyromitra splendida Raitv. - FCE 4: 30 (1974).

+ Haplotrichum parmastoi G. Langer [ as 'parmastii'] - FCE 33: 63 (1998).

* Heterodermia albopruinosa (Kurok.) Kurok. FCE 32: 21 (1998).

* Heterodermia allardii (Kurok.) Trass - FCE 29: 6 (1992). 
* Heterodermia arsenei (Kurok.) Kurok. - FCE 32: 21 (1998).

* Heterodermia cubensis (Kurok.) Trass - FCE 29: 10 (1992).

* Heterodermia cyathiformis (Kurok.) Kurok. FCE 32: 22 (1998).

* Heterodermia fragilissima (Kurok.) Trass FCE 29: 12 (1992).

* Heterodermia fauriei (Kurok.) Kurok. - FCE 32: 22 (1998).

* Heterodermia hypoleuca (Ach.) Trevis var. divergens Trass - FCE 37: 101 (2001) [2000].

* Heterodermia intermedia Trass - FCE 29: 23 (1992).

* Heterodermia isidiophora (Nyl.) D.D. Awasthi var. coralligera Trass - FCE 37: 102 (2001) [2000].

* Heterodermia isidiosa (Kurok.) Kurok. - FCE 32: 23 (1998).

* Heterodermia kurokawae Trass - FCE 29: 14 (1992).

* Heterodermia lamelligera (Taylor) Trass - FCE 29: 15 (1992).

* Heterodermia multiciliata (Kurok.) Trass - FCE 29: 16 (1992).

* Heterodermia obesa (Pers.) Trass - FCE 29: 16 (1992).

* Heterodermia pacifica (Kurok.) Kurok. - FCE 32: 23 (1998).

* Heterodermia palpebrata (Taylor) Trass - FCE 29: 17 (1992).

* Heterodermia pandurata (Kurok.) Trass - FCE 29: 17 (1992).

* Heterodermia peruviana (Kashiw. \& Kurok.) Kurok. - FCE 32: 23 (1998).

* Heterodermia polyrhiza (Kurok.) Kurok. - FCE 32: 24 (1998).

* Heterodermia rugulosa (Kurok.) Trass - FCE 29: 19 (1992).

* Heterodermia spinigera (Kurok.) Trass - FCE 29: 19 (1992) [nom. inval., Art. 34.1].

* Heterodermia spinigera (Kurok.) Kurok. - FCE 32: 24 (1998).

* Heterodermia spinulosa (Kurok.) Trass - FCE 29: 20 (1992).

* Heterodermia subascendens (Asahina) Trass - FCE 29: 20 (1992).

* Heterodermia subcomosa (Nyl.) Trass - FCE 29: 20-21 (1992).

* Heterodermia subisidiosa (Kurok.) Kurok. FCE 32: 24 (1998).

* Heterodermia trichophora (Kurok.) Trass - FCE 29: 21 (1992).
* Heterodermia trichophoroides (Kurok.) Kurok. - FCE 32: 24 (1998).

* Heterodermia tropica (Kurok.) Kurok. - FCE 32: 24 (1998).

+ Hyalopeziza arctica Raitv. - FCE 23: 5 (1985).

+ Hyalopeziza glacialis Raitv. - FCE 12: 3 (1981).

+ Hyalopeziza hexagona (Fuckel) Raitv. - FCE 9: 1 (1977).

+ Hyalopeziza latispora Raitv. - FCE 12: 4 (1981).

+ Hyalopeziza schachdarica Raitv. - FCE 12: 4 (1981).

+ Hyalopeziza scrupulosa (P. Karst.) Raitv. - FCE 9: 1 (1977).

+ Hydropus flocculinus Kalamees - FCE 26: 7 (1987).

+ Hymenochaete allantospora Parmasto FCE 37: 58 (2001) [2000].

+ Hymenochaete americana Greslebin \& Parmasto - FCE 37: 59 (2001) [2000].

+ Hymenochaete burdsallii Parmasto - FCE 37: 61 (2001) [2000].

+ Hymenochaete cinnamomea (Pers.) Bres. subsp. spreta (Peck) Parmasto - FCE 37: 62 (2001) [2000].

+ Hymenochaete gigasetosa Parmasto-FCE 40: 41 (28 Jan 2004) [2003].

+ Hymenochaete indica Parmasto - FCE 40: 42 (28 Jan 2004) [2003].

+ Hymenochaete jobii Parmasto - FCE 37: 62 (2001) [2000].

+ Hymenochaete reticulata Parmasto - FCE 37: 64 (2001) [2000].

+ Hymenochaete ryvardenii Parmasto - FCE 37: 65 (2001) [2000].

+ Incrupila alatavica Raitv. - FCE 12: 5 (1981).

+ Incrupila kondarensis Raitv. - FCE 12: 5 (1981).

+ Incrupila narynica Raitv. - FCE 12: 5-6 (1981).

+ Inocybe ampullaceocystidiata Shchukin FCE 23: 1 (1985).

+ Inocybe hygrophoroides Shchukin - FCE 23: 2 (1985).

+ Inocybe oblectabilis P.D. Orton var. odora Shchukin - FCE 23: 2 (1985).

+ Inocybe ravaensis Kalamees \& Shchukin FCE 23: 4 (1985).

+ Inonotopsis Parmasto - FCE 2: 12 (4 Jan 1973). 
+ Inonotopsis subiculosa (Peck) Parmasto - FCE 2: 12 (4 Jan 1973).

+ Irpex oreophilus (Lindsey \& Gilb.) Niemelä FCE 33: 95 (1998).

+ Junghuhnia brownii (Humb.) Niemelä - FCE 33: 95 (1998).

+ Lachnellula angustispora Raitv. - FCE 9: 3 (1977).

+ Lachnellula minuscula Raitv. - FCE 9: 3 (1977).

+ Lachnum alnifolium (Raitv.) Raitv. - FCE 20: 1 (1986).

+ Lachnum altaicum (Raitv.) Raitv. - FCE 20: 1 (1986).

+ Lachnum clavigerum (Svrček) Raitv. - FCE 17: 3 (1985).

+ Lachnum dasiphorae (Raitv.) Raitv. - FCE 20: 1 (1986).

+ Lachnum fagicolum (W. Phillips) Raitv. - FCE 20: 1 (1986).

+ Lachnum fuscidulum (Cooke) Raitv. - FCE 20: 1 (1986).

+ Lachnum hissaricum (Raitv. \& Faizova) Raitv. - FCE 20: 1 (1986).

+ Lachnum ledi (Raitv.) Raitv. - FCE 20: 2 (1986).

+ Lachnum lespedezae (Raitv.) Raitv. - FCE 20: 2 (1986).

+ Lachnum palearum (Desm.) Raitv. - FCE 17: 3 (1985).

+ Lachnum pendulae Raitv. - FCE 37: 89 (2001) [2000].

+ Lachnum perplexum (Boud.) Raitv. - FCE 20: 2 (1986).

+ Lachnum ponticum (Raitv.) Raitv. - FCE 20: 2 (1986).

+ Lachnum pseudocannabinum (Raitv.) Raitv. FCE 17: 3 (1985).

+ Lachnum pudicelloides (Raitv.) Raitv. - FCE 17: 3 (1985).

+ Lachnum rubi (Bres.) Raitv. - FCE 17: 3 (1985).

+ Lachnum sinegoricum (Raitv.) Raitv. - FCE 17: 3 (1985).

+ Lachnum soppittii (Massee) Raitv. - FCE 20: 2 (1986).

+ Lachnum sulphurellum (Peck) Raitv. - FCE 20: 2 (1986).

+ Lachnum tenuissimum (Quél.) Raitv. - FCE 17: 3 (1985).

+ Lachnum turkestanicum (Raitv.) Raitv. - FCE 20: 2 (1986).

+ Lachnum vantschense (Raitv.) Raitv. - FCE 20: 2 (1986)
+ Lachnum virtembergense (Matheis) Raitv. FCE 17: 4 (1985).

+ Laetiporus cincinnatus (Morgan) Burds., Banik \& T.J. Volk - FCE 33: 13 (1998).

+ Lasiobelonium stipitiatum Raitv. - FCE 12: 6 (1981).

+ Lasiobelonium subfuscum Raitv. - FCE 12: 7 (1981).

* Lecanora swartzii (Ach.) Ach. ssp. soralifera E.S. Hansen - FCE 44: 44 (2008).

* Leciophysma parvum Øvstedal - FCE 48: 65 (2011).

+ Lepista juniperini Kalamees - FCE 26: 4 (1987).

+ Lepista saeva (Fr.) P.D. Orton var. anserina (Fr.) Kalamees \& A.I. Ivanov - FCE 30: 29 (1992).

* Massalongia griseolobulata Øvstedal - FCE 47: 45 (2010).

+ Melanoleuca brunnea Kalamees - FCE 26: 5 (1987).

+ Melanoleuca pallidicutis Bresinsky - FCE 42: 1 (2006).

+ Melanoleuca zaaminensis Kalamees - FCE 26: 6 (1987).

+ Melaspilea galligena Zhurb. \& I. Zhdanov - FCE 50: 92 (2013).

+ Mollisia minima Raitv. \& Kutorga - FCE 39: 30 (2003) [2002].

+ Mycena pura (Pers.) P. Kumm. f. roseobrunnescens Kalamees - FCE 26: 7 (1987).

+ Naetrocymbe kentrospora (Branth) Alstrup FCE 46: 14 (2009).

* Neuropogon sphacelatus (R. Br.) Alstrup \& E.S. Hansen - FCE 37: 12 (2001) [2000].

+ Oliveonia citrispora (Hauerslev) P. Roberts FCE 33: 129 (1998).

+ Oliveonia nodosa (Hauerslev) P. Roberts - FCE 33: 129 (1998).

+ Oliveonia termitophila (Oberw. \& Ryvarden) P. Roberts - FCE 33: 130 (1998).

+ Oliveoniaceae P. Roberts - FCE 33: 128 (1998).

+ Oliveorhiza P. Roberts - FCE 33: 128 (1998).

+ Oliveorhiza anapauxilla P. Roberts - FCE 33: 129 (1998).

+ Omphalina fuliginea Kalamees - FCE 26: 3 (1987).

+ Omphalina psammophila Shchukin - FCE 18: 5 (1985).

\# Opegrapha trassii S.Y. Kondr. \& Coppins - FCE 32: 10 (1998). 
+ Panaeolus atrobalteatus Pegler \& A. Henrici - FCE 33: 105 (1998).

+ Phaeohelotium hylocomnii Raitv. \& Leenurm - FCE 37: 87 (2001) [2000].

* Phaeophyscia primaria (Poelt) Trass - FCE 15: 2 (1981).

* Phaeophyscia sulphurascens (Zahlbr.) Trass - FCE 15: 2 (1981).

+ Phellinus betulinus (Murrill) Parmasto - FCE 43: 41 (2007).

+ Phellinus betulinus (Murrill) Parmasto ssp. orienticus Parmasto - FCE 43: 41 (2007).

+ Phellinus gilbertsonii M.J. Larsen - FCE 37: 52 (2001) [2000].

+ Phellinus lonicericola Parmasto - FCE 38: 59 (2001).

+ Phialina flaveola (Cooke) Raitv. - FCE 9: 1 (1977).

+ Phialina montana Raitv. - FCE 23: 5 (1985).

+ Phialina obscura Raitv. - FCE 23: 6 (1985).

+ Phialina pseudopuberula (Graddon) Raitv. FCE 9: 1 (1977).

+ Phialina viridiflavescens (Rehm) Raitv. - FCE 9: 1 (1977).

+ Phialoscypha Raitv. - FCE 8: 1 (1977).

+ Phialoscypha lachnobrachya (Desm.) Raitv. FCE 8: 2 (1977).

+ Phialoscypha lachnobrachyoides Raitv. FCE 8: 2 (1977).

+ Phialoscypha spiraeaicola Raitv. - FCE 8: 3 (1977).

+ Phlebia acanthocystis Gilb. \& Nakasone FCE 33: 85 (1998).

+ Phlebia brunneofusca (Hjortstam \& Ryvarden) Nakasone \& Gilb. - FCE 33: 88 (1998).

+ Phlebia subfascicularis (Wakef.) Nakasone \& Gilb. - FCE 33: 85 (1998).

\# Polycoccum follmannii (C.W. Dodge) Alstrup FCE 39: 1 (2003) [2002].

+ Polyporus choseniae (Vassilkov) Parmasto [as 'chozeniae'] - FCE 5: 35 (1974).

+ Proprioscypha echinulata Raitv. \& Leenurm - FCE 37: 89 (2001) [2000].

+ Psathyrella badhyzensis Kalamees - FCE 15: 7 (1981).
+ Psathyrella gramina Kalamees - FCE 27: 7 (1989).

+ Pseudotomentella ochracea Kõljalg \& E. Larss. - FCE 33: 54 (1998).

+ Pyrenopeziza euphrasiae (Fuckel) Kuntze var. brevispora Raitv. - FCE 39: 33 (2003) [2002].

+ Radulodon pseudomucidus (Petch) Stalpers FCE 33: 137 (1998).

+ Radulodon subvinosus (Berk. \& Broome) Stalpers - FCE 33: 137 (1998).

+ Scutellinia caucasica Kullman \& Raitv. FCE 10: 6 (1978).

+ Scutellinia heterosculpturata Kullman \& Raitv. - FCE 7: 4 (1977).

+ Smarodsia Raitv. \& Vimba - FCE 42: 98 (2006).

+ Smarodsia stollii Raitv. \& Vimba - FCE 42: 98 (2006).

+ Stalpersia Parmasto - FCE 38: 51 (2001).

+ Stalpersia orientalis Parmasto - FCE 38: 51 (2001).

* Sticta tesselata Øvstedal - FCE 47: 46 (2010).

* Szczawinskia phylicae Øvstedal - FCE 47: 47 (2010).

* Tephromela rimosula Øvstedal - FCE 47: 47 (2010).

* Thelotrema tristanense Øvstedal - FCE 47: 48 (2010).

+ Tricholoma frondosae Kalamees \& Shchukin - FCE 38: 14 (2001).

+ Tricholoma ulvinenii Kalamees - FCE 38: 18 (2001).

+ Tricholomopsis osiliensis Vauras - FCE 45: 87 (2009).

+ Trichopezizella heteropilosa Raitv. - FCE 12: 7 (1981).

+ Trichopezizella paradoxa Raitv. - FCE 12: 7-8 (1981).

+ Trichopezizella vaasmae Raitv. - FCE 23: 6 (1985).

+ Tyromyces sibiricus Penzina \& Ryvarden FCE 33: 109 (1998).

+ Unguiculella lupini Raitv. - FCE 9: 2 (1977). 
132 Folia Cryptog. Estonica 